\title{
Morphological transformations in Brazilian peripheral areas: a case study of the Vila Cabral neighborhood at Campina Grande-PB
}

XXIV International Conference

of the Iberoamerican Society

of Digital Graphics

Medellin | Colombia

\author{
Jaqueline Brandão \\ Universidade Federal da Paraíba | Brasil | jaquelinebrandaao@gmail.com \\ Frederico Ribeiro Costa \\ Universidade Federal da Paraíba | Brasil | frederico.costa@arquitetura.ufjf.br \\ Geovany Silva \\ Universidade Federal da Paraíba | Brasil | galexarq.ufpb@gmail.com
}

\begin{abstract}
This article describes an analysis of the morphological changes between 2005 and 2020 in the Vila Cabral neighborhood, located on the outskirts of the Brazilian city of Campina Grande. The research was developed in two different analytical scenarios, adopting the algorithmicparametric computational implementation as a methodology, and applying the following procedures: (i) formal decomposition of a sample of the urban fabric; (ii) visibility graph analysis (VGA); (iii) analysis of the diversity of uses and (iv) analysis of urban density (populational and built). The study demonstrated that the association of different urban analysis tools strengthens decision-making in the context of evidence-based urban design.
\end{abstract}

Keywords: Algorithmic-parametric urban analysis; Evidence-based design; Urban morphology; Campina Grande; Brazilian peripheral sprawl.

\section{INTRODUÇÃO}

O presente artigo apresenta uma síntese de trabalhos desenvolvidos no campo do projeto urbano, mais especificamente relacionados à qualidade e à avaliação do ambiente construído na escala da cidade, vinculados ao grupo de pesquisa Desempenho e Inovação Aplicados ao Projeto (DIAProj CNPq), da Universidade Federal da Paraíba, e que possuem como processo de estudo (i) elaboração de uma análise da forma urbana e dos aspectos da ocupação territorial (que se traduzem nos paradigmas de dispersão / compactação urbana); e (ii) atuação no suporte à tomada de decisões, com ênfase na reabilitação dos espaços urbanos e na promoção de cidades mais sustentáveis face aos principais aspectos da compacidade urbana, da diversidade de usos e do planejamento urbano orientado ao desempenho.

Objeto de estudo aplicado nesta pesquisa, o município de Campina Grande está localizado no interior do estado da Paraíba (Figura 1), distante $125 \mathrm{~km}$ ao oeste da capital João Pessoa, e com uma população de 409,7 mil habitantes (IBGE, 2019). Implantada no semiárido brasileiro, sua posição geográfica privilegiada entre o sertão e litoral paraibano fez da cidade um importante polo regional econômico, industrial e educacional. No entanto, sua influência econômica e a sua capacidade de ofertar bens e serviços transformou a cidade em um núcleo atrativo para ação de agentes sociais produtores do espaço urbano, principalmente aqueles que operam sobre a influência do capital privado, articulando estratégias mais lucrativas para seus empreendimentos por meio da promoção da especulação imobiliária, concentrando os seus investimentos na periferia, regiões que sabidamente possuem menor valor de mercado (Costa, 2013; Lima, 2018; Maia, 2013).

De acordo com a definição apresentada por Correa (2004), os agentes sociais que influenciam na forma urbana podem ser classificados como: (i) os proprietários dos meios de produção dos setores industrial, comercial e de serviço; (ii) os proprietários fundiários que buscam ampliar a renda fundiária de suas propriedades com a especulação e comércio das terras rurais mediante a transição do espaço rural para o urbano; (iii) os promotores imobiliários que podem atuar em diversos âmbitos como incorporação, financiamento, estudo de viabilidade técnico-construtiva, construção de imóveis e comercialização; (iv) o Estado, que atua como promotor imobiliário ao fomentar subsídios voltados para moradia, promove a infraestrutura do espaço urbano e elabora as legislações urbanísticas vinculadas ao uso do solo; e (v) os grupos sociais excluídos, que compreendem a população de baixa renda sem acesso à moradia.

Já no entendimento de Capel (2013), todos os cidadãos atuam e usufruem da cidade, entretanto, somente alguns têm o poder de interferir na forma urbana: os agentes urbanos. De acordo com sua proposta de classificação, esses agentes podem ser (i) proprietários das terras; (ii) os proprietários dos meios de produção; (iii) os promotores imobiliários; e (iv) o Estado. O autor ainda destaca que, em geral, os cidadãos são categorizados apenas como atores 
sociais, ainda que estejam envolvidos em algum grau com os movimentos sociais urbanos.

Neste sentido, fica evidente que a produção socioespacial dentro do modelo capitalista é consequência da ação de um conjunto de agentes dotados de interesses específicos. Tratando especificamente dos processos de urbanização no município de Campina Grande, Maia (2010) aponta a existência de eixos de expansão urbana na cidade, caracterizando um movimento de dispersão da malha urbana para as regiões periféricas. De acordo com a autora, esse processo tem chamado atenção do setor imobiliário, incentivando a especulação de terrenos, principalmente após a concretização de empreendimentos robustos, a exemplo da construção de supermercados de grandes redes, centros universitários, e condomínios fechados horizontais (Corrêa, 2011; Costa, 2013; Maia, 2013).

À vista do contexto exposto, esta investigação tem por objetivo principal realizar uma abordagem quantiqualitativa do processo de transformações formais e espaciais ocorridas na região periférica do perímetro urbano do município de Campina Grande, estado da Paraíba - Brasil, mais especificamente àquelas sucedidas no bairro Vila Cabral (Figura 2), uma região caracterizada, predominantemente, por ocupações autoconstruídas irregulares (Maia, 2013). O estudo lança mão do conceito de evidence-based design (Hamilton \& Watkins, 2008; Mourelatos \& Zhou, 2006), com a análise aqui proposta podendo balizar e oferecer suporte às decisões projetuais do espaço edificado. A hipótese tomada por este estudo é que a edificação do centro universitário da Faculdade de Ciências Sociais Aplicadas (UNIFACISA) implantada no ano de 2005 e o inícios das obras do empreendimento Heron Marinho Business \& Living, no ano de 2011, atuaram como equipamentos-polo de atração para o crescimento urbano do entorno imediato dessas edificações. Isso potencializou as transformações urbanas no bairro e acentuou as modificações nos aspectos de uso e ocupação do solo, promovendo também um aumento no padrão de dispersão após o início de outros investimentos imobiliários privados, como os novos condomínios verticais e horizontais.

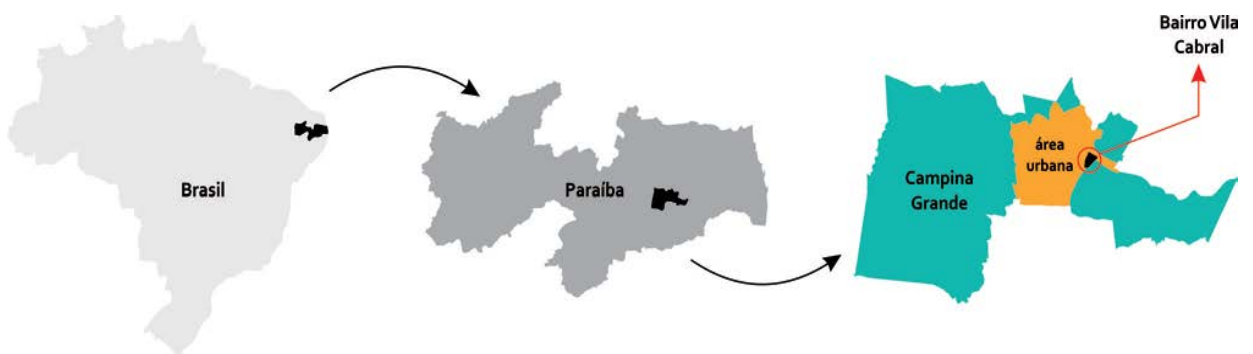

Figura 1: Identificação do objeto de estudo, o bairro Vila Cabral, situado no município de Campina Grande / Paraíba, Brasil.

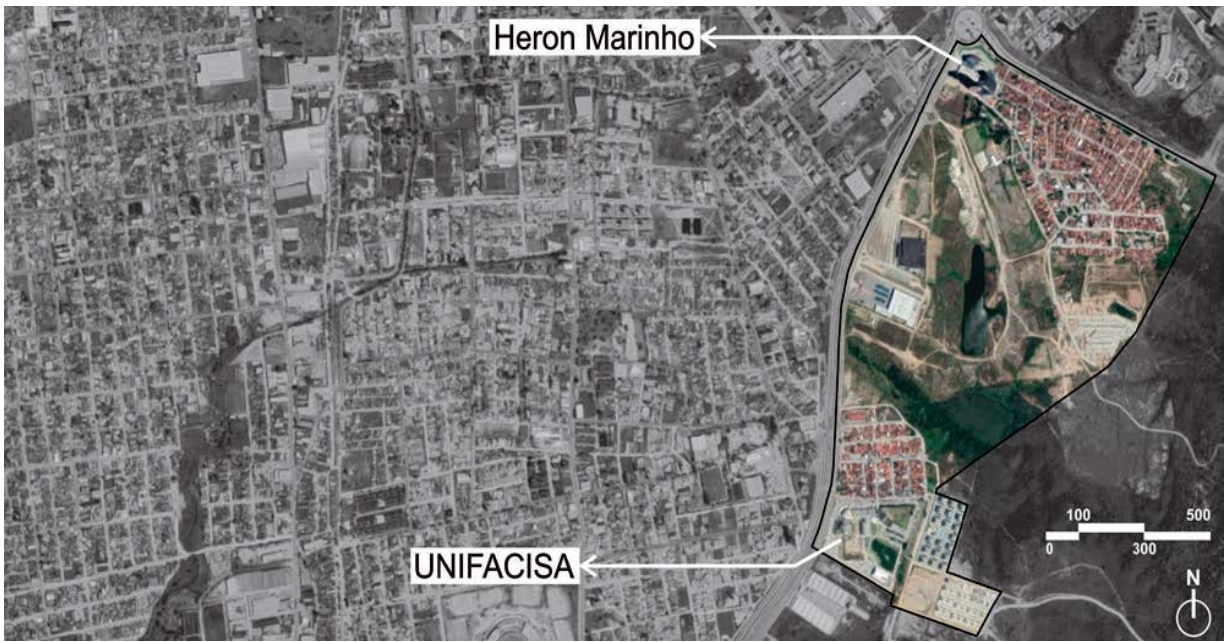

Figura 2: O tecido urbano e o entorno imediato do bairro Vila Cabral no ano de 2020. Na figura estão identificados os dois equipamentos-polos tomados pela hipótese da pesquisa, o Heron Marinho Business \& Living e o Centro Universitário da UNIFACISA.

COMPACIDADE, DISPERSÃO E DESENHO URBANO: UMA BREVE REVISÃO

No contexto de diversas pesquisas (Lima et al., 2019; Nechyba \& Walsh, 2004; Netto \& Krafta, 2009; Newman \& Kenworthy, 2000), há uma clara proposta de reflexão sobre os impactos que as propriedades morfológicas - ou formais do espaço urbano - exercem na qualidade do espaço urbano, com destaque para os problemas advindos das ações propostas pelo atual modelo de produção urbana no Brasil, que estimula o mal funcionamento das cidades e constitui um risco aos principais aspectos da urbanidade. Neste sentido, Netto \& Krafta (2009) apontam 
que o padrão de crescimento urbano no Brasil, país como um processo de industrialização tardio, ocorre principalmente de duas maneiras distintas: (i) por meio do adensamento de áreas interurbanas, promovendo um processo de verticalização possivelmente excessivo; (ii) por meio da dispersão periférica, aumentando os limites da malha urbana.

Ambos modelos, analisados a luz do arcabouço teórico que fundamenta esta pesquisa, podem ser considerados problemáticos e, de acordo com os apontamentos de um extenso rol de autores, têm contribuído para o aumento significativo no custo da gestão e da manutenção das infraestruturas urbanas, impactando no consumo de recursos naturais e influenciando na dinâmica das cidades, já que impõem dificuldades a mobilidade pedonal e aumentam a dependência das pessoas por meios de transportes convencionais (Farr, 2013; Gehl, 2013; Netto \& Krafta, 2009; Rogers, 2001).

A dispersão impacta em diversos aspectos da qualidade de vida urbana, em especial na saúde pública (Ewing et al., 2003; Frumkin et al., 2004; Frumkin, 2002). Os problemas decorrentes do transporte e do trânsito consequente da baixa densidade, crescente número de automóveis e autopistas (Brueckner, 2000; Nechyba \& Walsh, 2004; Squires, 2002), assim como impactos na poluição por crescente emissão de gases estufa e derivados combustíveis (Bereitschaft \& Debbage, 2013), têm sido alguns dos temas recorrentes nas pesquisas urbanas em países mais ricos e nos países em desenvolvimento.

Por sua vez, o desenho urbano e planejamento pode atuar na reabilitação urbana e na dependência automotiva consequente destes modelos (Newman \& Kenworthy, 1989 e 2006; Kenworthy \& Laube, 1996). Correntes de contraposição a este modelo de dispersão e impacto urbano-ambiental foram variadas no campo da sustentabilidade urbana, propondo conjuntos mais compactos, multipolarizados, passíveis de locomoção pendular por meios alternativos de transporte, e com maior oferta de usos, residências e equipamentos por área quadrada urbanizada (Duany \& Plater-Zyberk, 1992; Frey 2003; Newman \& Kenworthy, 2000; Rueda, 1997; Talen, 2011). Assim, a ideia de uma cidade mais densa, compacta, caminhável, com bons espaços públicos e verdes, surgem como alternativa de uma cidade de metabolismo urbano circular e de menor pegada ambiental. Entende-se, portanto, que a forma urbana passa a ser uma condicionante importante para cidades mais sustentáveis (Jabareen, 2006; Jenks et al., 2005; Jenks et al., 2000).

\section{PROCEDIMENTOS METODOLÓGICOS}

A investigação aqui pormenorizada teve como principal elemento de abordagem prática a associação entre três diferentes abordagens do espaço urbano, para assim construir um processo de análise formal e espacial, buscando compreender as transformações no tecido urbano por meio de aspectos visuais e de atributos objetivos. Em relação aos procedimentos metodológicos aplicados no âmbito desta investigação (Figura 3), foi implementada uma análise quanti-qualitativa do bairro Vila Cabral (objeto de estudo aplicado), realizando: (i) uma decomposição do tecido urbano a partir de uma amostra homogênea (Coelho, 2013); (ii) a elaboração de mapas de visibilidade; (iii) uma análise objetiva da diversidade de usos (aplicação de índices de uso misto); (iv) da densidade urbana construída e da densidade populacional (líquida e bruta). Estes índices e indicadores foram coletados e analisados por meio de recursos computacionais algorítmico-paramétricos, a partir de componentes algorítmicos do plugin Grasshopper para o software Rhinoceros3D, organizados para realização desta pesquisa, além da incorporação de duas ferramentas do sistema CityMetrics, desenvolvido por Lima (2017): os clusters de nome "Spacematrix" e "MXI".

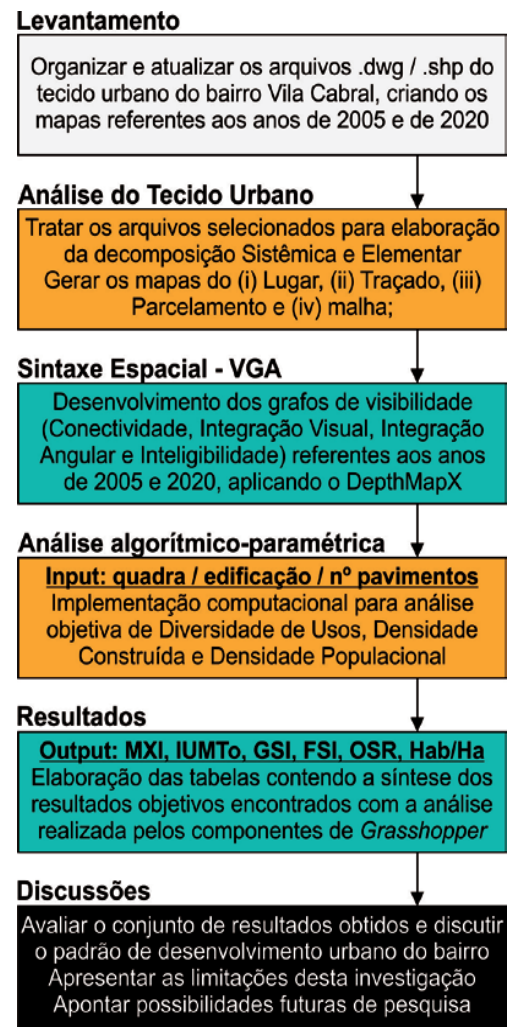

Figura 3: Síntese do percurso metodológico e dos procedimentos realizados no contexto desta investigação.

Neste seguimento, com o objetivo de proporcionar uma rápida visualização dessas transformações, foram construídos, em ambiente digital, dois cenários distintos relativos aos anos de 2005 e de 2020, adotando como amostra o limite administrativo do bairro Vila Cabral (determinado pela prefeitura municipal de Campina Grande), que contém o sítio onde foi construído o empreendimento Heron Marinho Business \& Living, no ano de 2011. Foram acrescidos na amostra o terreno onde foi construído o centro universitário da UNIFACISA, em 2005, e os terrenos que hoje constituem dois novos condomínios verticais, construídos nos anos de 2014 e em 2017.

Desta forma, os procedimentos metodológicos realizados podem ainda ser separados em três etapas distintas, a saber: (i) o estudo da forma urbana do bairro, por meio da aplicação do método de Decomposição Sistêmica e da 
Decomposição Elementar do tecido urbano, apresentado por Coelho (2013); (ii) uma análise de grafos de visibilidade (Visibility Graph Analysis - VGA), proposta por Turner et al. (2001) como alternativa metodológica para investigar as relações da configuração urbana sob a Lógica Social do Espaço (Hillier \& Hanson, 1984), implementando a software DepthMapX (versão 0.50) para analisar aspectos da Sintaxe Espacial (e.g. medidas de conectividade, de integração visual, integração angular, de inteligibilidade e uma análise de fluxos); (iii) implementação computacional para suporte no cálculo objetivo de relações da forma urbana (diversidade de usos, densidade construída e a densidade populacional), por meio da lógica algorítmicaparamétrica (Lima, Costa \& Rosa, 2020).

Na terceira etapa, após a introdução dos componentes Grasshopper, se dá a implementação do índice de mistura urbana Mixed-use Index (MXI), desenvolvido por Hoek (2008), além da análise da diversidade de usos considerando todos os pavimentos verticalizados, por meio da implementação de um dos clusters de CityMetrics para cálculo do IUMTo (Silva, 2016). Por fim, é desenvolvida uma implementação do cluster de CityMetrics que permite uma análise dos indicadores de densidade construída Spacematrix, propostos por Berghauser Pont \& Haupt (2010).

\section{ÍNDICES DE MISTURA URBANA: MXI E IUMTo}

$\mathrm{O}$ índice de uso misto (Mixed-use Index - MXI), elaborado por Hoek (2008), tem por objetivo estabelecer uma relação entre o somatório de área construída residencial e não residencial ao nível da rua (pavimento térreo), medindo objetivamente a diversidade de usos em uma determinada amostra. De acordo com Hoek (2008), quanto mais essa relação entre as áreas se aproximar do equilíbrio (50/50), maior será a mistura urbana e, consequentemente, maior será a diversidade de usos.

Já o índice de mistura urbana IUMTo, proposto por Silva (2016), atua estabelecendo a mesma relação entre o total de áreas construídas residenciais e não residenciais que o índice MXI, entretanto o IUMTo pondera sobre o somatório total das áreas de cada edificação, o que também inclui na análise todos os seus pavimentos acima do térreo (nível da rua), permitindo verificar o equilíbrio/mistura dos usos considerando o nível de verticalização da amostra.

\section{INDICADORES DE DENSIDADE CONSTRUÍDA SPACEMATRIX}

Para realização desta análise, foram utilizados três indicadores fundamentais propostos por Berghauser Pont \& Haupt (2010): (i) Total do solo ocupado (Ground Space Index - GSI; (ii) Aproveitamento vertical nas áreas em que o solo é ocupado (Floor Space Index - FSI); e (iii) proporção de espaços abertos (Open Space Ratio - OSR). O GSI representa uma relação entre a amostra e as projeções das edificações no solo, podendo ser obtido por meio de uma razão entre área total e o somatório das áreas do solo que se encontram ocupadas por edificações. Já o FSI representa uma média de quanto as projeções das edificações contidas na amostra são aproveitadas, podendo ser obtido por meio da divisão entre a área construída total (considerando também os pavimentos verticalizados) e a área da amostra. O OSR pode ser obtido por meio de uma divisão entre a área total de solo ocupado por edificações e a área construída total.

\section{RESULTADOS}

Ao realizar a decomposição formal do tecido urbano (Figura 4), verificou-se que o Vila Cabral possui dois principais eixos de crescimento urbano, o que estimula a consolidação de duas regiões distintas dentro do bairro, desconectadas devido as características topográficas da região. A Decomposição Sistêmica também evidenciou que ambos os eixos se localizam próximos as duas edificações tomadas como ímãs do desenvolvimento urbano na hipótese deste estudo, tornando perceptível, portanto, a influência que os dois empreendimentos exerceram no incentivo ao espraiamento da região.

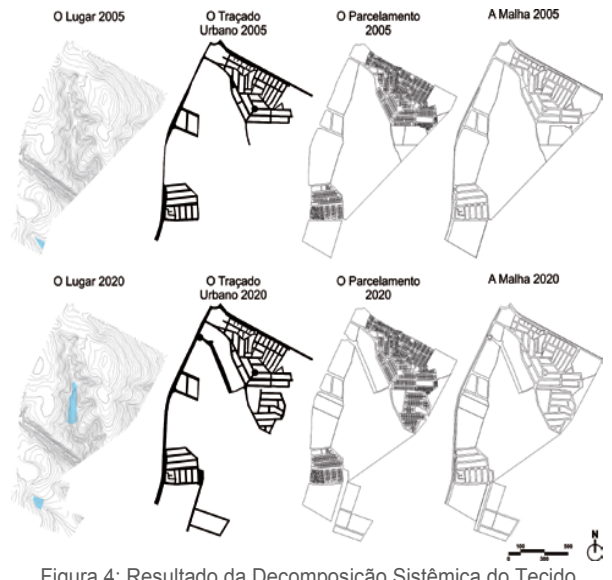

Figura 4: Resultado da Decomposição Sistêmica do Tecido Urbano no bairro Vila Cabral, apresentada graficamente dividida em dois cenários: 2005 e 2020. Esta figura pode ser acessada em tamanho maior no link https://imgur.com/wjQMSN9

Seguindo com a aplicação da metodologia desenvolvida por Coelho (2013), foi elaborado em seguida uma Decomposição Elementar na amostra homogênea do tecido urbano do bairro Vila Cabral, identificando seus elementos: (i) Quadra (Figura 5 e Figura 6); (ii) Rua (Figura 6); (iii) Edifício Comum (Figura 7); Edifício Singular em 2005 (Figura 8); e Edifício Singular em 2020 (Figura 9). Após a decomposição destes elementos, a escala menor tornou possível verificar com mais detalhes o processo de crescimento urbano no bairro, verificando, por exemplo, quais as novas edificações que passaram a ocupar a quadra, como essas edificações compõem a paisagem da rua, qual o Edifício Comum (edifício característico que representa a tipologia predominante na amostra) e quais os Edifícios Singulares (edifícios que destoam do Edifício Comum).

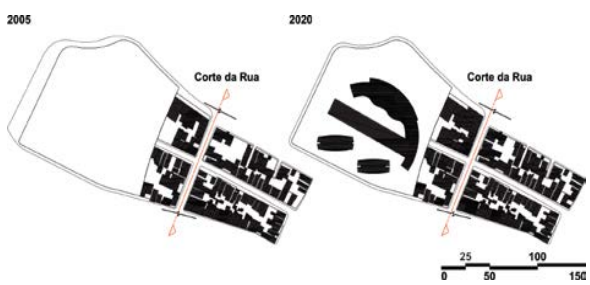

Figura 5: Decomposição Elementar da Quadra, representada graficamente nos dois cenários: 2005 e 2020 . Esta figura pode ser acessada em tamanho maior no link https://imgur.com/33FN3Ut 


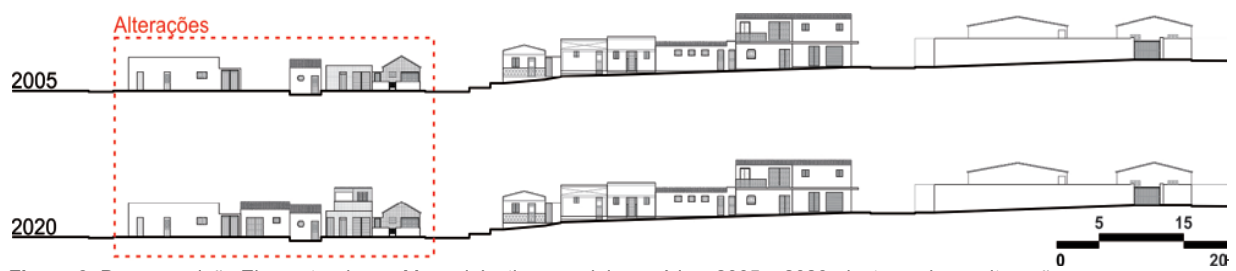

Figura 6: Decomposição Elementar da rua Manoel Justino nos dois cenários: 2005 e 2020, destacando as alterações.

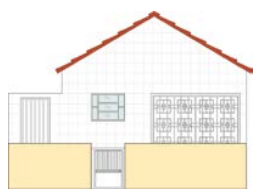

Figura 7: Decomposição Elementar do edifício comum nos dois cenários: habitação unifamiliar de um pavimento.

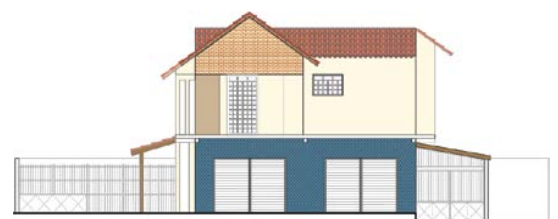

Figura 8: Decomposição Elementar do edifício singular identificado no cenário de 2005: habitação unifamiliar com comercio local no pavimento térreo.

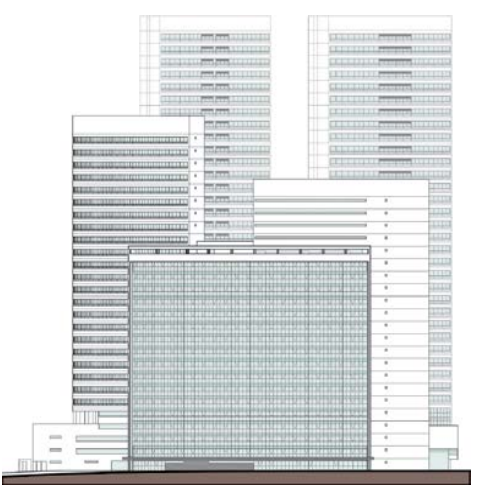

Figura 9: Decomposição Elementar do edifício singular identificado no ano de 2020: torre multiuso com 30 pavimentos.

Introduzindo a análise sintática (Figura 10), os mapas foram gerados a partir da análise de gráficos de visibilidade (Visibility Graph Analysis - VGA). Para o processamento dos mapas de ambos recortes temporais foi adotado um grid de 4 pixels, e para o cálculo da medida de integração a partir das relações de visibilidade, foram consideradas as medidas globais com o valor do raio = "n".

Mediante a leitura da escala cromática referente ao ano de 2005 , verificou-se que a área mais conectada visualmente do sistema está localizada na rotatória situada nas proximidades da área norte do bairro Vila Cabral, sendo também o ponto que apresenta um maior fluxo de agentes. Os mapas de Integração Visual e de Integração Angular expõe maior visibilidade em diferentes trechos da Avenida Senador Argemiro de Figueiredo, representado pela escala cromática que tende para o tom vermelho (cores mais quentes), ramificando-se em tons de laranja e verde para as principais vias de acesso aos bairros circunvizinhos. Verificou-se também que tanto a parte norte quanto a parte sul do bairro apresentam um baixo grau de visibilidade, representado por uma escala cromática de tons que tendem para o azul (cores mais frias), situação que é evidenciada pelo baixo valor de Inteligibilidade $\left(\mathrm{R}^{2}=0,26\right)$, pela contagem de nós igual a 109.853 e pela profundidade média igual a 2,86 para o sistema (Figura 11).

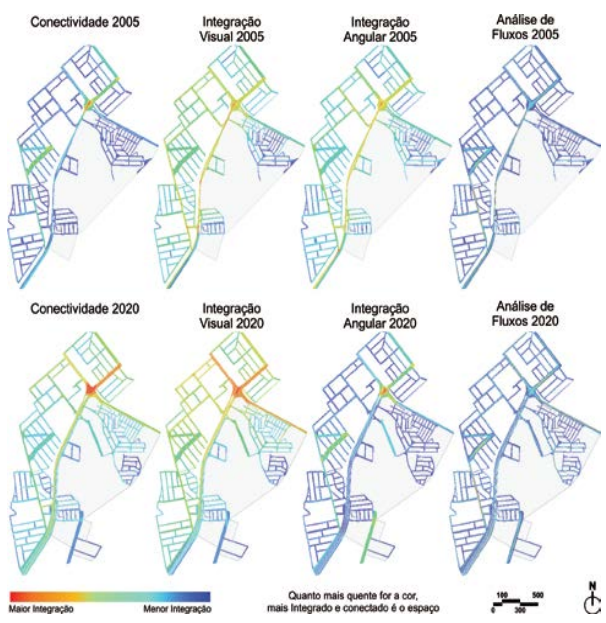

Figura 10: Mapas sintáticos elaborados para análise de visibilidade. Esta figura pode ser acessada em tamanho maior no link https://imgur.com/HrR7t1X

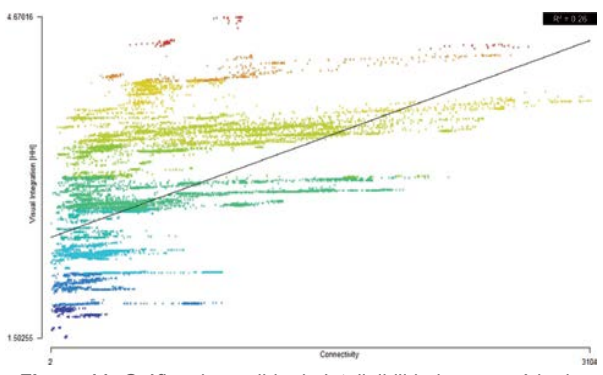

Figura 11: Gráfico da medida de Inteligibilidade no cenário de 2005. Esta figura pode ser acessada em tamanho maior no link https://imgur.com/84Axw5v

No que tange o ano de 2020, a área de maior conectividade visual também se concentra na rotatória situada nas proximidades da parte superior do bairro Vila Cabral. Os mapas de integração visual e integração angular apresentam uma maior concentração de cores quentes (que convergem para o vermelho) especificamente no início da Avenida Prefeito Bezerra Severino Cabral, ramificando-se em graus intermediários de visibilidade 
(tons de laranja e verde) para a rua Engenheiro José Celino Filho, BR-230 e Avenida Senador Argemiro de Figueiredo, principais vias de acesso aos bairros circunvizinhos, onde também se concentra um maior fluxo de agentes.

Comparando os resultados obtidos nos dois cenários, nas áreas menos integradas do bairro, ocorre uma significativa variação das cores nas áreas que tendem para o azul, demonstrando que no ano de 2020 estas áreas passaram a ser mais integradas visualmente. No que diz respeito à inteligibilidade (Figura 12), a medida foi reduzida $\left(R^{2}=\right.$ $0,16)$, o que pode ser justificado pela expansão das áreas periféricas do bairro, tendo como contagem de nós igual a 168.025 e a profundidade média igual a 3,65 para 0 sistema.

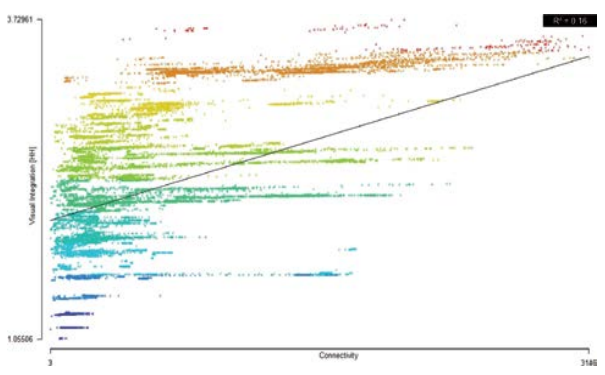

Figura 12: Gráfico da medida de Inteligibilidade no cenário de 2020. Esta figura pode ser acessada em tamanho maior no link https://imgur.com/AD3bL8I
ANÁLISE OBJETIVA DA DIVERSIDADE DE USOS E DAS DENSIDADES CONSTRUÍDA E POPULACIONAL

Após a implementação dos componentes Grasshopper desenvolvidos para esta investigação e das duas ferramentas de CityMetrics que foram adotadas para realização da análise algorítmico-paramétrica (Figura 13), foram obtidas informações gerais relativas aos dois cenários (Tabela 1), além de serem encontrados os valores objetivos para os indicadores MXI, IUMTo, GSI, FSI, OSR e a densidade populacional em habitantes por hectare (hab/ha) referentes aos os dois cenários estudados (Tabela 2), o que possibilitou uma comparação dos resultados e uma compreensão das alterações ocorridas no Vila Cabral.

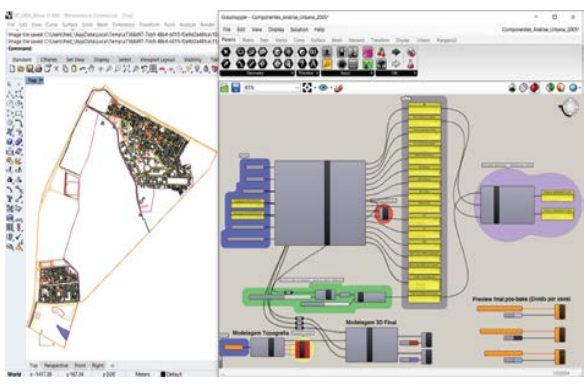

Figura 13: Análise algorítmica-paramétrica (Rhinoceros3D e Grasshopper) de um cenário em andamento no bairro Vila Cabral. Esta figura pode ser acessada em tamanho maio no link https://imgur.com/LFuz5eO

Tabela 1: Informações gerais dos elementos que compõem o tecido urbano do bairro Vila Cabral, dispostos para permitir a comparação das transformações ocorridas entre os anos de 2005 e de 2020.

\begin{tabular}{|c|c|c|c|c|c|c|c|c|c|}
\hline \multicolumn{10}{|c|}{ Informações gerais - bairro Vila Cabral } \\
\begin{tabular}{|c|c|c|c|c|c|c|c|c|c|}
\hline \multicolumn{2}{|c|}{ Área de análise (Ha) } & Área das quadras (Ha) & \multicolumn{2}{|c|}{ Número de quadras } & \multicolumn{2}{|c|}{ Número de lotes } & Número de edificações \\
\hline $\mathbf{2 0 0 5}$ & $\mathbf{2 0 2 0}$ & $\mathbf{2 0 0 5}$ & $\mathbf{2 0 2 0}$ & $\mathbf{2 0 0 5}$ & $\mathbf{2 0 2 0}$ & $\mathbf{2 0 0 5}$ & $\mathbf{2 0 2 0}$ & $\mathbf{2 0 0 5}$ & $\mathbf{2 0 2 0}$ \\
\hline 105,15 & 122,63 & 29,73 & 47,71 & 57 & 72 & 1325 & 1550 & 1263 & 1702 \\
\hline
\end{tabular}
\end{tabular}

Tabela 2: Síntese dos resultados objetivos dos aspectos da diversidade de usos, da densidade construída e da densidade populacional por hectare, calculados nos dois cenários (2005 e 2020).

\begin{tabular}{|c|c|c|c|c|c|c|c|c|c|}
\hline \multicolumn{10}{|c|}{ Índices dimensionais do espaço urbano no bairro Vila Cabral } \\
\hline \multicolumn{5}{|c|}{ Cenário referente ao ano de 2005} & \multicolumn{5}{|c|}{ Cenário referente ao ano de 2020} \\
\hline \multicolumn{2}{|c|}{ Diversidade de usos } & \multicolumn{3}{|c|}{ Densidade construída } & \multicolumn{2}{|c|}{ Diversidade de usos } & \multicolumn{3}{|c|}{ Densidade construída } \\
\hline $\mathrm{MX}$ & IUMTo & GSI & FSI & OSR & $\mathrm{MXI}$ & IUMTo & GSI & FSI & OSR \\
\hline $80 \%$ res. & $70 \%$ res. & \multirow{2}{*}{0,17} & \multirow{2}{*}{0,22} & \multirow{2}{*}{0,75} & $72 \%$ res. & $64 \%$ res. & \multirow{2}{*}{0,21} & \multirow{2}{*}{0,43} & \multirow{2}{*}{0,49} \\
\hline $20 \%$ ñ-res. & $30 \%$ ñ-res. & & & & $28 \%$ ñ-res & $36 \%$ ñ-res. & & & \\
\hline \multicolumn{5}{|c|}{ Densidade populacional estimada } & \multicolumn{5}{|c|}{ Densidade populacional estimada } \\
\hline Bruta & Líquida & \multicolumn{3}{|c|}{ Total de habitantes na área } & Bruta & Líquida & \multicolumn{3}{|c|}{ Total de habitantes na área } \\
\hline 53 & 93 & \multicolumn{3}{|c|}{5.601} & 109 & 165 & \multicolumn{3}{|c|}{13.368} \\
\hline
\end{tabular}

A Tabela 1 apresenta os dados gerais relativos aos recortes temporais estabelecidos. A amostra referente ao ano de 2005 apresenta uma área total de 105,15 hectares, sendo 29,73 hectares ocupados por um total de 57 quadras. As quadras, por sua vez, possuem um total de 1325 lotes e 1263 edificações. Já no cenário de 2020, a amostra homogênea apresenta uma área total de 122,63 hectares, sendo que 47,71 hectares são ocupados por um total de 72 quadras. Ao todo são 1550 lotes e um total de 1702 edificações.

Os resultados objetivos que foram encontrados após a implementação da abordagem algorítmica-paramétrica, sistematizados na Tabela 2, evidenciaram que a área de estudo é predominantemente residencial em ambos os cenários, considerando o nível da rua, representado por um índice de uso misto (MXI) igual a $80 \%$ das edificações construídas sendo residenciais e $20 \%$ não-residenciais para o ano e 2005 , e $72 \%$ residenciais e $28 \%$ nãoresidenciais para o ano de 2020. Ainda em relação a diversidade de usos, os resultados obtidos para a variedade dos usos no total da área construída, ou seja, considerando todos os pavimentos verticais (IUMTo), mostram que a região apresenta $70 \%$ da área construída composta por edificações residências e $30 \%$ composta por edificações não-residenciais para o ano de 2005, e $64 \%$ da área construída composta por edificações residências e $36 \%$ composta por edificações não-residenciais para o ano de 2020. 
Comparando os resultados obtidos nos dois cenários, pode-se afirmar que em relação aos indicadores de densidade construída (Figura 14), a área de estudo apresenta uma pequena variação na ocupação do solo (GSI), subindo de 0,17 em 2005 para 0,21 no ano de 2020 . Já em relação ao aproveitamento do solo (FSI), o indicie praticamente dobrou, saindo de 0,22 no ano de 2005 para 0,43 , no ano de 2020. Entretanto é possível afirmar que este aumento considerável no indicie de verticalização é influenciado empreendimento Heron Marinho Business \& Living (identificado como Edifício Singular em 2020 na decomposição elementar), composto por uma torre empresarial de 20 pavimentos, duas torres residenciais de 30 pavimentos, uma torre com 130 flats distribuídos em 22 pavimentos, além de um hotel com 150 apartamentos. Em relação ao indicie de espaços abertos (OSR), a tabela 2 aponta que houve uma diminuição de 0,75 para 0,49 , o que pode ser justificado pelo o aumento da ocupação do solo e da área construída total.

Em relação a densidade populacional, a área de estudo continha 5.601 habitantes em 2005, com densidade bruta de 53 hab/ha e liquida de 93 hab/ha. Já no ano de 2020, estima-se uma população total de 13.368 pessoas, um aumento significativo, representado por uma densidade bruta igual a 109 hab/ha e líquida de 165 hab/ha.
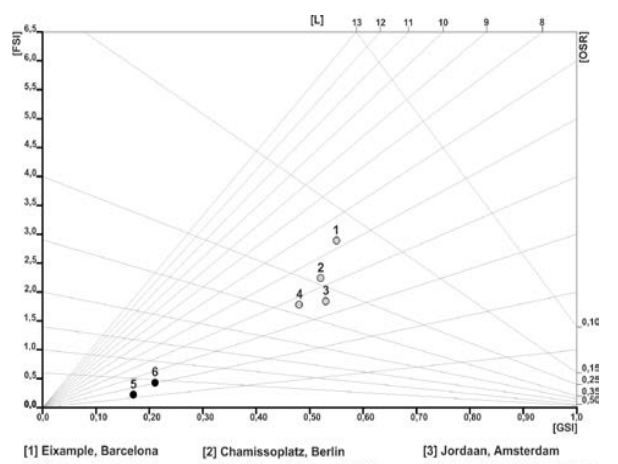

[4] De Pijp. Amsterdam [5] Vila Cabral, C. Grande, 2005 [6] Vila Cabral, C. Grande, 2020

Figura 14: Gráfico de Matriz Espacial (Spacematrix), indexando os dois cenários e outras quatro cidades analisadas por

Berghauser Pont \& Haupt (2010). Esta figura pode ser acessada em tamanho maior no link https://imgur.com/GjCtW7O

\section{DISCUSSÕES}

Os resultados encontrados estimulam a discussão acerca dos processos de projeto do espaço urbano, mais especificamente, aqueles que se relacionam com o padrão de dispersão e crescimento urbano em áreas periféricas do Brasil, abordando três aspectos relevantes da forma urbana relacionados ao contexto do desenho urbano sustentável: (i) diversidade de usos; (ii) compacidade urbana; e (iii) integração espacial. Foi possível validar a hipótese inicial da pesquisa, evidenciando que a implantação dos dois empreendimentos privados (Centro Universitário da UNIFACISA e Heron Marinho Business \& Living) potencializaram o crescimento do bairro Vila Cabral e do seu entorno imediato, na medida em que as transformações viárias aumentaram a conexão do bairro com o restante da cidade, consequentemente atraindo novos investimentos que modificaram sua configuração formal. Porém, mesmo com um aumento nos índices dimensionais investigados, é válido destacar que os aspectos qualitativos de desenho urbano e a própria infraestrutura do bairro Vila Cabral ainda são bastante deficitárias no cenário de 2020. Assim, apesar de melhorias nos índices dimensionais, a qualidade dos espaços e dos projetos ali implantados ainda deixam muito a desejar. A questão ambiental também é outro ponto relevante, visto que a diminuição na disposição de áreas permeáveis e verdes, de forma não ordenada, pode impactar drasticamente na absorção de água pelo solo, ou até mesmo agravar as condições de conforto ambiental do bairro para os próximos anos, em uma região de muito calor e déficit de chuva (semiárido do Nordeste Brasileiro).

O método de análise lançado por esta investigação, baseado na associação entre diferentes procedimentos metodológicos (Decomposição Sistêmica, Decomposição Elementar, Visibility Graph Analysis - VGA, e implementação computacional algorítmico-paramétrica), demonstrou eficiência, podendo ser aplicado no suporte à tomada de decisões em um contexto de evidence-based design, auxiliando as análises e facilitando a compreensão das transformações por meio de valores objetivos (métricas) e de mapas visuais (decomposições e grafos VGA).

Como limitações desta pesquisa, identifica-se que a adoção do limite administrativo do bairro como amostra homogênea do tecido urbano não é a melhor opção para determinar a área de análise. Existem relações espaciais que transcendem o perímetro administrativo determinado pelo município, algo que ficou evidente na decomposição sistêmica do bairro: existem duas zonas distintas dentro do Vila Cabral. Sendo assim, a adoção de um processo metodológico para delimitar o perímetro de estudo por meio de relações espaciais seria mais frutífero. Também é fundamental introduzir novas abordagens, especialmente no âmbito qualitativo, entendendo que a compreensão dos aspectos da qualidade urbana só pode ser obtida por meio de análises em várias escalas.

Neste senso, para os estudos futuros é possível que os procedimentos de coleta e de análise de dados sejam aperfeiçoados, ampliando o escopo para mais bairros, inserindo aspectos de conforto ambiental aplicado especificamente aos estudos da forma urbana. Em tempos de pandemia e alto contágio por coronavírus (e outras possíveis doenças regionais), muitas das quais são transmitidas pelo ar, refinar os procedimentos analíticos com ênfase nos espaços abertos e ventilados para quadras urbanas e edifícios será um grande desafio para as regiões quentes e secas do Brasil, como é o caso da cidade de Campina Grande e de diversas outros municípios implantados no Nordeste do país. É importante destacar que a associação metodológica proposta é passiva de aperfeiçoamentos, permitindo abordagens mais robustas sob a ótica da Lógica Social do Espaço, permitindo ainda que novas ferramentas algorítmicoparamétricas sejam introduzidas, articulando novos parâmetros relacionados a diferentes paradigmas de projeto urbano, admitindo que o escopo da pesquisa seja expandido para avaliar outros objetos de estudo.

\section{AGRADECIMENTOS}

Agradecemos ao Comitê Executivo Internacional da SIGraDi por toda assistência oferecida, bem como aos revisores deste trabalho por suas contribuições precisas. 
Ao Conselho Nacional de Desenvolvimento Científico e Tecnológico (CNPq), ao Programa de Pós-graduação em Arquitetura e Urbanismo da Universidade Federal da Paraíba (PPGAU UFPB) e aos laboratórios Desempenho e Inovação Aplicados ao Projeto (DIAPRoj CNPq) e Laboratório de Investigação em Arquitetura e Urbanismo (DOMVS CNPq), pela estrutura e pelo suporte contínuo. O presente trabalho foi realizado com apoio da Coordenação de Aperfeiçoamento de Pessoal de Nível Superior - Brasil (CAPES) - Código de Financiamento 001.

\section{REFERÊNCIAS}

Bereitschaft, B. \& Debbage, K. (2013). Urban form, air pollution, and $\mathrm{CO}^{2}$ emissions in large US metropolitan areas. The Professional Geographer, 65(4), 612-635.

Berghauser Pont, M. \& Haupt, P. (2010) Spacematrix: Space, Density and Urban Form. Rotterdam: NAI Publishers.

Brueckner, J. K. (2000). Urban sprawl: diagnosis and remedies. International Regional Science Review, 23(2), 160-171.

Coelho, C. D. (2013). Os elementos urbanos. Lisboa: Argumentum.

Correa, R. L. (2004). O Espaço Urbano. São Paulo: Ática.

Costa, L. B. (2013). Estruturação da cidade de Campina Grande: As estratégias e intencionalidades do mercado imobiliário. (Dissertação de Mestrado) Universidade Federal da Paraíba, João Pessoa. Disponível em: <http://www.ccen.ufpb.br/ppgg/contents/documentos/disserta coes/leonardo_barboza.pdf>. Acesso em: 15 mar. 2020.

Duany, A. \& Plater-Zyberk, E. (1992). The second coming of the American small town. Wilson Quarterly, 16(1), 3-51.

Ewing, R., Schmid, T., Killingsworth, R., Zlot, A. \& Raudenbush, S. (2003). Relationship between urban sprawl and physical activity, obesity, and morbidity. American Journal of Health Promotion, 18(1), 47-57.

Farr, D. (2013) Urbanismo Sustentável: desenho urbano com a natureza. Porto Alegre: Bookman.

Frey, H. (2003). Designing the city: Towards a more sustainable urban form. Londres: Taylor \& Francis.

Frumkin, H. (2002). Urban sprawl and public health. Public Health Reports. 117(3), 201-217.

Frumkin, H., Frank, L. \& Jackson, R. J. (2004). Urban sprawl and public health: Designing, Planning, and Building for Healthy Communities. Washington: Island Press.

Gehl, J. (2013). Cidades para Pessoas. São Paulo: Perspectiva.

Hamilton, D. K. \& Watkins, D. H. (2008). Evidence-based design for multiple building types. Nova Jersey: John Wiley \& Sons.

Hillier, B. \& Hanson, J. (1984). The social logic of space. Nova lorque: Cambridge University Press.

Hoek, J. W. (2008) Towards a Mixed-use Index (MXI) as a tool for urban planning and analysis. Disponível em: http://joostvandenhoek.com/mediapool/80/805179/data/PhD_ proceedings_2009_layout_v10_spreads_hoek.pdf. Acesso em: 14 mar. 2020.

IBGE - Instituto Brasileiro de Geografia e Estatística - 2019: Disponivel em: <https://cidades.ibge.gov.br/brasil/pb/campina -grande/panorama>. Acesso em: 06/04/2020.

Jabareen, Y. R. (2006). Sustainable urban forms: Their typologies, models, and concepts. Journal of Planning Education and Research, 26(1), 38-52.

Jenks, M., Burgess, M. J. R., Acioly, C., Allen, A., Barter, P. A., \& Brand, P. (2000). Compact cities: sustainable urban forms for developing countries. Londres: Taylor \& Francis.
Jenks, M. \& Dempsey, N. (2005). Future forms and design for sustainable cities. Londres: Routledge.

Kenworthy, J. R. \& Laube, F. B. (1996). Automobile dependence in cities: an international comparison of urban transport and land use patterns with implications for sustainability. Environmental Impact Assessment review, 16(4-6), 279-308.

Lima, A. M. 2018. Condomínios horizontais fechados e o uso do solo urbano em Campina Grande - PB (2000-2017). (Dissertação de Mestrado) Universidade Federal do Rio Grande do Norte, Natal. Disponivel em: <https://repositorio.ufrn.br/jspui/bitstream/123456789/24984/ 1/AdjaelMaracajaDeLima_DISSERT.pdf>. Acesso em: 19 mar. 2020

Lima, F. T. A., Montenegro, N., Paraízo, R. C., Kós, J. R. (2019) Citymetrics: sistema (para)métrico para análise e otimização de configurações urbanas. Oculum Ensaios, 16(2), 409-427.

Lima, F. T. A., Costa, F. R. \& Rosa, A. (2020). Lógica algorítmicaparamétrica e urbanismo: uma revisão teórica e de modelos computacionais para projetos urbanos. Gestão \& Tecnologia de Projetos, 15(2), 84-97.

Maia, D. S. (2010). A periferização e a fragmentação da cidade: loteamentos fechados, conjuntos habitacionais populares e loteamentos irregulares na cidade de Campina Grande-PB, Brasil. Scripta Nova, 14(331), paper 80.

Maia, D. S., Cardoso, C. A. A., Alonso, S. F \& Silva, R. B. S. (2013). As desigualdades socioespaciais expressas no habitar. In D. Elias, M. E. B. Sposito \& B. R. Soares (Eds.). Agentes econômicos e reestruturação urbana regional Campina Grande e Londrina (117-138). São Paulo: Outras Expressões.

Mourelatos, Z. P. \& Zhou, J. (2006). A design optimization method using evidence theory. Journal of Mechanical Design, 128(4), 901-908.

Nechyba, T. J. \& Walsh, R. P. (2004). Urban sprawl. Journal of Economic Perspectives, 18(4), 177-200.

Netto, V. M. \& Krafta, R. (2009). A forma urbana como problema de desempenho: o impacto de propriedades espaciais sobre o comportamento urbano. Revista Brasileira de Estudos Urbanos e Regionais, 11(2), 157-180.

Newman, P. G., \& Kenworthy, J. R. (1989). Cities and automobile dependence: an international sourcebook. Brookfield: Gower Publishing.

Newman, P. \& Kenworthy, J. (2000). Sustainable urban form: the big picture. In E. Burton, M. Jenks \& K. Williams (Eds.). Achieving Sustainable Urban Form, (109-120). Londres: Spon Press.

Newman, P. \& Kenworthy, J. (2006). Urban design to reduce automobile dependence. Opolis, 2(1), 35-52.

Rogers, R. (2001). Cidades para um Pequeno Planeta. Barcelona: Gustavo Gili.

Rueda, S. (1997). La ciudad compacta y diversa frente a la conurbación difusa. Ciudades para un futuro más sostenible, 19(01), 69-80.

Silva, G. J. A. (2016). A sustentabilidade aplicada ao Projeto de Arquitetura e Urbanismo. Relatório de Pós-doutorado. Universidade de Lisboa, Faculdade de Arquitetura.

Squires, G. D. (2002). Urban sprawl: Causes, consequences, \& policy responses. Washington: Urban Insitute Press.

Talen, E. (2011). Sprawl retrofit: sustainable urban form in unsustainable places. Environment and Planning B: Planning and Design, 38(6), 952-978.

Turner, A., Doxa, M., O'Sullivan, D. \& Penn, A. (2001). From Isovists to Visibility Graphs: a methodology for the analysis of architectural space. Environment and Planning B, 28(1), 103121. 